\title{
Cardioneuroablation for the treatment of vagally mediated atrial fibrillation and vasovagal syncope
}

\author{
Aleksandra Wróblewska', Antoni Wileczek², Agnieszka Reichert ${ }^{3}$, Sebastian Stec² \\ 'Department of Cardiology, Specialistic Hospital, Tarnów, Poland \\ 2Division of Cardiac Electrophysiology, Cardioneuroablation, Ablation and Pacing, Subcarpathian Centre of Cardiovascular Interventions, Department of Cardiology, \\ Sanok, Poland \\ ${ }^{3}$ Department of Cardiology, Regional Specialist Hospital, Stalowa Wola, Poland
}

\author{
Correspondence to: \\ Aleksandra Wróblewska, MD, \\ Department of Cardiology, \\ Specialistic Hospital, \\ Szpitalna 13 \\ 33-100 Tarnów, Poland, \\ phone: +48 1463105 26, \\ e-mail: \\ wroblewska.ola@gmail.com \\ Copyright by the Author(s), \\ 2021 \\ Kardiol Pol. 2021; \\ 79 (10): 1151-1152 \\ DOI: 10.33963/KP.a2021.0073 \\ Received: \\ May 18, 2021 \\ Revision accepted: \\ July 22, 2021 \\ Published online: \\ July 22, 2021
}

Autonomic nervous system activation can induce multifarious changes in atrial electrophysiology and provoke atrial tachyarrhythmias, such as atrial tachycardia and vagally mediated atrial fibrillation (VM-AF) [1, 2]. This type of atrial fibrillation (AF) was defined as paroxysmal AF that occurs following vagal activation in such situations as after a big meal, during sleep, or in response to other recognized vagal triggers. Episodes of AF may be induced by neurally mediated reflex syncope (NMRS). Cardioneuroablation (CNA), a relatively novel technique based on percutaneous radiofrequency ablation of the vagal ganglia, can be used in patients with NMRS and VM-AF $[3,4]$.

A 33-year-old man was admitted to the hospital for AF ablation and CNA. He reported repeated episodes of syncope and palpitation since the age of 15 . Syncope was preceded by prodromal symptoms, such as weakness, dizziness, and palpitation. Several 24-hour Holter monitoring tests revealed sinus bradycardia (mean heart rate, 40-44 beats per minute [bpm]; minimum heart rate, 28-30 bpm; the longest RR interval, $2.8 \mathrm{~s}$ [sinus arrest]). A 12lead electrocardiogram (ECG) showed sinus bradycardia and inverted $T$ waves in leads $\mathrm{V}_{3}-\mathrm{V}_{5}$. Echocardiography revealed no abnormalities. A positive tilt test response was classified as type $2 \mathrm{~B}$ (cardioinhibition with asystole) according to the modified Classification by the Vasovagal Syncope International Study (Figure $1 \mathrm{~A}, \mathrm{~B}$; Supplementary material, Figure 1S). Based on the current guidelines and tilt test results, VM-AF, NMRS, and bradycardia-tachycardia syndrome were diagnosed. The patient was referred for pulmonary vein isolation as well as modified anatomically-guided binodal and biatrial CNA with bilateral extracardiac vagal nerve stimulation (ECANS) according to Pachon et al. [3]. Prior to CNA and pulmonary vein isolation (PVI), detailed electrophysiology study (EPS) excluded atrioventricular nodal reciprocating tachycardia and atrioventricular reentry tachycardia. Additionally, ultrasound-guided ECANS was performed from the nonsubcranial region of the internal jugular vein, which showed repetitive sinus asystole and complete atrioventricular block for 8 seconds during AF. Proximal coronary sinus stimulation caused recurrent AF. Sinus rhythm was restored with electrical cardioversion. Pulmonary vein isolation was followed by endocardial ablation at 5 anatomic sites of the ganglionated plexi in the left and right atria, mapped according to the modified Pachon's method (Figure 1C, D). Left and right ECANS postprocedure did not elicit cardioinhibition or arrhythmia (AF or ventricular tachycardia). Sinus rhythm increased from 55-60 bpm to 85-90 bpm. Following atropine administration, resting sinus rhythm increased from 87 to $92 \mathrm{bpm}$ at 10 minutes ( $\leq 6 \%)$. On the control tilt test at 1 month, nitroglycerine administration resulted in presyncope with nausea (Supplementary material, Figure 2S). Heart rhythm and blood pressure decreased. Syncope was not observed. Intravenous atropine administration ( $1.5 \mathrm{mg}$ ) led to an increase in resting sinus rhythm from 61 to $82 \mathrm{bpm}$ at 10 minutes (34\%). Holter ECG monitoring at 6 weeks revealed sinus rhythm with a mean heart rate of $68 \mathrm{bpm}$ (53-90 bpm) without arrhythmia.

There is growing evidence that AF can be triggered by vagal hyperactivity [3, 4]. Cardioneuroablation is an effective and safe treatment for vasovagal syncope and can be used during AF ablation. There are some limitations 


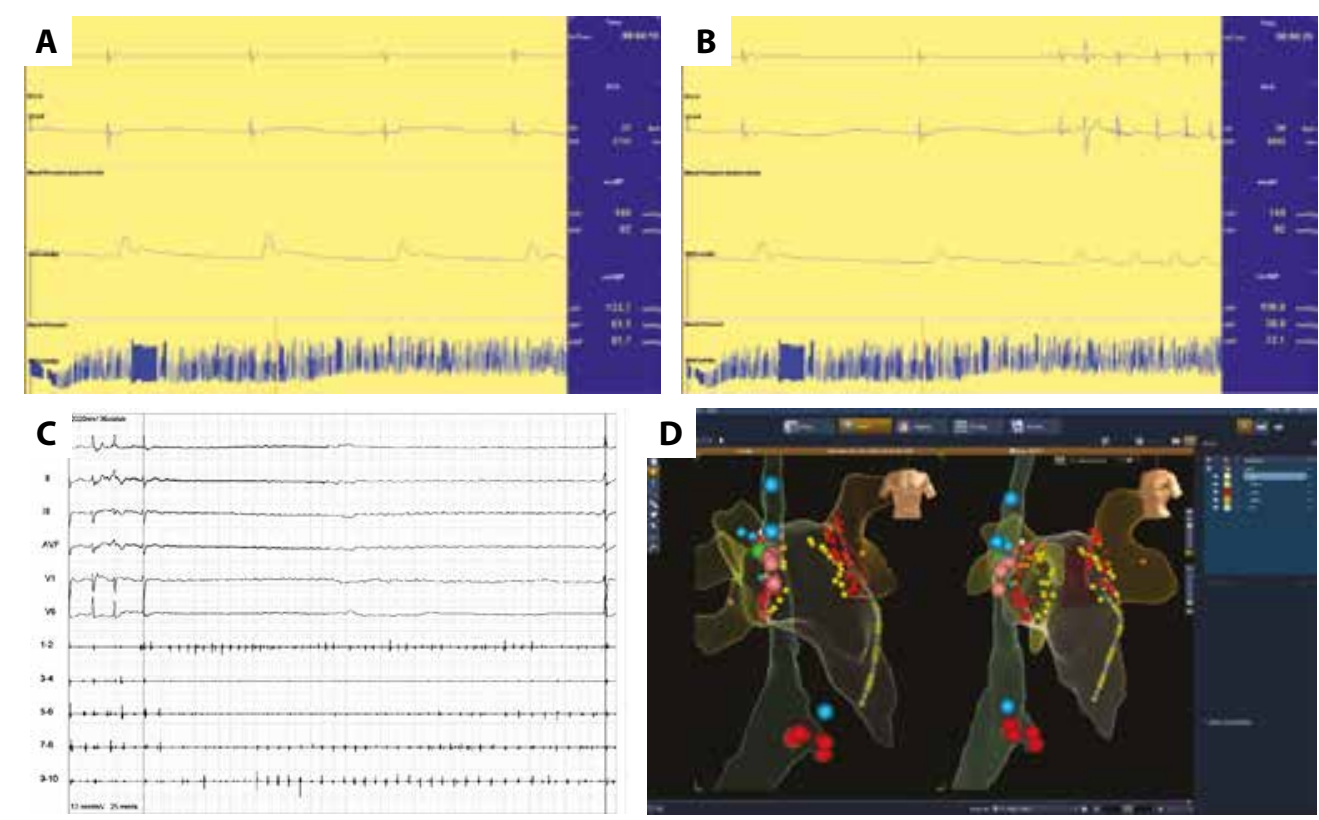

Figure 1. A-D. Noninvasive continuous beat-to-beat blood pressure monitoring during head-up tilt test in a patient with syncope. Nitroglycerin administration ( $0.4 \mathrm{mg}$ sublingually), loss of consciousness and syncope; a decrease in sinus rhythm to $20 \mathrm{bpm}$, followed by 4 seconds of sinus asystole was documented. A. A decrease in sinus rhythm induced atrial fibrillation. B. Vagally mediated atrial fibrillation occurred after table lowering; then, within 3 minutes, AF resolved with symptomatic sinus arrest lasting 2 seconds. C. Surface ECG and diagnostic bipolar potentials recorded with a diagnostic decapolar catheter located in the CS 9-10 being in the proximal CS. Extracardiac vagal nerve stimulation generated an artifact on surface ECG (bold line) and ventricular asystole lasting 8 seconds, with no effect on atrial rhythm. D. Left-hand side, anteroposterior view; right-hand side, left anterior oblique projection. Large blue dots in the upper part represent right phrenic nerve stimulation sites in the SVC. A single blue dot in the lower part represents His-Bundle pacing. Small red and yellow dots represent ablation sites during pulmonary vein isolation and GP ablation on the anterior and posterior left atrial wall, respectively. Large pink dots represent GP ablation sites in the SVC. A yellow decapolar catheter is placed in the coronary sinus

Abbreviations: CS, coronary sinus; ECG, electrocardiogram; GP, ganglionated plexi; SVC, superior vena cava

to our management due to the lack of preprocedural atropine test. However, non-invasive tests prior to inducibility of AF excluded neuropathy. Previously described atropine test (post-CNA) showed a significant increase in heart rhytm. The long-term clinical utility of partial innervation vs. complete denervation, as shown by atropine test, as well as tilt test, was not fully validated, especially during follow-up. Although clinical follow-up is excellent and baseline mean heart rhythm remains higher, simultaneous performance of PVI and CNA cannot confirm the superiority of one of these techniques for AF. Moreover, the current standards for AF prevention require $\mathrm{PVI}$, therefore both techniques were simultaneously applied $[3,5]$.

\section{Supplementary material}

Supplementary material is available at https://journals. viamedica.pl/kardiologia_polska.

\section{Article information}

Conflict of interest: $\mathrm{SS}$ is author of several patents and share holder of Medicine S.A. No products were used in this case.

Open access: This article is available in open access under Creative Common Attribution-Non-Commercial-No Derivatives 4.0 International (CC BY-NC-ND 4.0) license, allowing to download articles and share them with others as long as they credit the authors and the publisher, but without permission to change them in any way or use them commercially. For commercial use, please contact the journal office at kardiologiapolska@ptkardio.pl.
How to cite: Wróblewska A, Wileczek A, Reichert A, et al. Cardioneuroablation for the treatment of vagally mediated atrial fibrillation and vasovagal syncope. Kardiol Pol. 2021; 79(10): 1151-1152, doi: 10.33963/KP.a2021.0073.

\section{REFERENCES}

1. Hindricks G, Potpara T, Dagres N, et al. ESC Scientific Document Group. 2020 ESC Guidelines for the diagnosis and management of atrial fibrillation developed in collaboration with the European Association for Cardio-Thoracic Surgery (EACTS): the task force for the diagnosis and management of atrial fibrillation of the European Society of Cardiology (ESC) developed with the special contribution of the European Heart Rhythm Association (EHRA) of the ESC. Eur Heart J. 2021; 42(5): 373-498, doi: 10.1093/eurheartj/ehaa612, indexed in Pubmed: 32860505.

2. Brignole M, Moya A, de Lange FJ, et al. 2018 ESC Guidelines for the diagnosis and management of syncope. Eur Heart J. 2018; 39(21): 1883-1948, doi: 10.1093/eurheartj/ehy037.

3. Pachon-M El, Pachon-Mateos JC, Higuti C, et al. Relation of fractionated atrial potentials with the vagal innervation evaluated by extracardiac vagal stimulation during cardioneuroablation. Circ Arrhythm Electrophysiol. 2020; 13(4): e007900, doi: 10.1161/CIRCEP.119.007900, indexed in Pubmed: 32188285.

4. Stec S, Dobaj $Ł$, Śledź A, et al. Cardioneuroablation for management of cardioinhibitory vasovagal syncope and pacemaker complications. HeartRhythm Case Rep. 2020; 6(8): 531-534, doi: 10.1016/j.hrcr.2020.04.021, indexed in Pubmed: 32817835.

5. Aksu T, Guler TE, Yalin K. There are still debates on cardioneuroablation strategy despite increasing evidence. Commentary to the article: „Cardioneuroablation using an anatomical approach: a new and promising method for the treatment of cardioinhibitory neurocardiogenic syncope". Kardiol Pol. 2019; 77(1): 65-66, doi: 10.5603/KP.2019.0010, indexed in Pubmed: 30672584. 